\title{
SO(3) homology of graphs and links
}

\author{
BENJAMIN COOPER \\ MATT HOGANCAMP \\ VYACHESLAV KRUSHKAL
}

\begin{abstract}
The SO(3) Kauffman polynomial and the chromatic polynomial of planar graphs are categorified by a unique extension of the Khovanov homology framework. Many structural observations and computations of homologies of knots and spin networks are included.
\end{abstract}

57M27; 57M25, 05C10, 05C31

\section{Introduction}

Mikhail Khovanov [11] introduced a categorification of the Temperley-Lieb algebra. Recently, the first two authors [4] showed that there are chain complexes within this construction that become the Jones-Wenzl projectors in the image of the Grothendieck group $\mathrm{K}_{0}$. These chain complexes are unique up to homotopy and idempotent with respect to the tensor product: $C \otimes C \simeq C$. It is now well-known (see Fendley and Krushkal [5]) that the chromatic algebra and the SO(3) Birman-Murakami-Wenzl algebra can be constructed using the second Jones-Wenzl projector. In this paper we use the formulation of Bar-Natan [1] to extend the original categorification of the Temperley-Lieb algebra to categorifications of the SO(3) BMW algebra and the chromatic algebra. Previous work of Helme-Guizon and Rong [9] and Stošić [15] on the categorification of the chromatic polynomial has been focused on constructions which are in many respects independent of structural choices such as the Frobenius algebra. In this paper we obtain an essentially unique categorification of the chromatic polynomial of planar graphs.

We begin by interpreting the second Jones-Wenzl projector in the Temperley-Lieb algebra over the ring of $q$-power series with $\mathbb{Z}$-coefficients:

$$
\left.p_{2}=\right)\left(-\frac{1}{q+q^{-1}} \asymp=\right)\left(+\sum_{i=1}^{\infty}(-1)^{i} q^{2 i-1} \asymp\right.
$$

This power series is replaced by a chain complex in the categorification which is then shown to satisfy uniqueness and idempotence properties up to homotopy. While the 
categorification of the Jones-Wenzl projectors $p_{n}$ for all $n$ is presented in [4], in this paper we give a self-contained account for the second projector. Using this chain complex the 2-categorical "canopolis" structure of the Khovanov categorification then extends from a categorification of the Temperley-Lieb planar algebra to a categorification of the $\mathrm{SO}(3) \mathrm{BMW}$ algebra and chromatic algebra. It is checked that the local relations in these algebras are satisfied up to homotopy by our construction.

We conclude with a number of calculations of homologies of links and spin networks and some preliminary observations about the structure of the space of morphisms. Two explicit calculations are included in order to demonstrate the ease with which our model lends itself to calculation. We include the chromatic homology for tree and generalized theta graphs. The homology of the sheet algebra is computed and we conjecture that all graph homology is structured in a specific way. Due to the universal nature of the construction in [4] the authors believe that these calculations will agree with those made using other frameworks for the categorification of representation theory.

\section{Diagrammatic algebras}

This section summarizes the relevant background on definitions of the Temperley-Lieb algebra, the chromatic algebra and the $\mathrm{SO}(3) \mathrm{BMW}$ algebra, and on the relations between them.

\subsection{Temperley-Lieb algebra}

The Temperley-Lieb algebra $\mathrm{TL}_{n}$ is the $\mathbb{Z}\left[q, q^{-1}\right]$-algebra determined by subjecting the generators $1, e_{1}, e_{2}, \ldots, e_{n-1}$ to the relations

(1) $e_{i} \cdot e_{j}=e_{j} \cdot e_{i}$ if $|i-j| \geq 2$

(2) $e_{i} \cdot e_{i \pm 1} \cdot e_{i}=e_{i}$

(3) $e_{i}^{2}=[2] e_{i}$

where the quantum integer [2] $=q+q^{-1}$.

Each generator $e_{i}$ can be pictured as a diagram consisting of $n$ chords between two collections of $n$ points on two horizontal lines in the plane. All strands are vertical except for two, connecting the $i-$ th and the $(i+1)-$ st points in each collection. For instance, when $n=3$ we have the following diagrams:

$$
1=|| \quad, \quad e_{1}=\bigcup \mid \text { and } \quad e_{2}=\mid \bigcup
$$


The multiplication is given by vertical composition of diagrams. Planar isotopy induces relations 1 and 2 between the generators above while the third relation states that a disjoint circle evaluates to $q+q^{-1}$.

This algebra is well-known in low-dimensional topology due to the extension from planar diagrams to tangles given by the Kauffman bracket relations:

$$
\aleph=q||-q^{2} \bigcap \text { and } \bigwedge^{\prime}=q^{-2} \bigcap-q^{-1}||
$$

$\mathrm{TL}_{n}$ is included into $\mathrm{TL}_{n+1}$ by adding a vertical strand on the right, and TL is defined to be $\bigcup_{n} \mathrm{TL}_{n}$. The trace $\operatorname{tr}_{\mathrm{TL}}: \mathrm{TL}_{n} \longrightarrow \mathbb{Z}\left[q, q^{-1}\right]$ is defined on the additive generators (rectangular pictures) by connecting the top and bottom endpoints by disjoint arcs in the complement of the rectangle in the plane. The result is a disjoint collection of circles in the plane, which are then evaluated by taking $\left(q+q^{-1}\right)^{\# \text { circles }}$.

2.2 Definition (Jones-Wenzl projector) There is a special element $p_{2} \in \mathrm{TL}_{2}$ (where the coefficients are taken to be rational functions of the variable $q$ ),

$$
p_{2}=1-\frac{1}{q+q^{-1}} e_{1},
$$

called the second Jones-Wenzl projector. Graphically,

$$
\stackrel{\downarrow}{\uparrow 1}=\mid-\frac{1}{q+q^{-1}} \bigcup
$$

The second Jones-Wenzl projector $p_{2}$ satisfies the properties

(1) $p_{2} \cdot e_{1}=0=e_{1} \cdot p_{2}$

(2) $p_{2} \cdot p_{2}=p_{2}$.

In representation theory, the Temperley-Lieb algebra is the algebra of $U_{q} \mathfrak{s u}(2)$-equivariant maps between $n$-fold tensor powers of the fundamental representation $V$ :

$$
\mathrm{TL}_{n}=\operatorname{Hom}_{U_{q} \mathfrak{s u}(2)}\left(V^{\otimes n}, V^{\otimes n}\right) .
$$

The subalgebra determined by the projector $p_{2}$ corresponds to the second irreducible

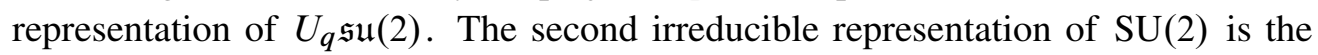
fundamental representation of $\mathrm{SO}(3)$. 


\subsection{The SO(3) BMW algebra}

We review some background material on the $\mathrm{SO}(N)$ Birman-Murakami-Wenzl algebra; see Birman and Wenzl [3] and Murakami [13] for more details. $\operatorname{BMW}(N)_{n}$ is the algebra of framed tangles on $n$ strands in $D^{2} \times[0,1]$ modulo regular isotopy and the $\mathrm{SO}(N)$ Kauffman skein relations:

$$
\begin{gathered}
Y-\lambda^{\prime}=\left(q^{2}-q^{-2}\right)(\mid-\bigcup) \\
\bigcap=q^{2(N-1)} \mid \text { and } Y=q^{2(1-N)}
\end{gathered}
$$

By a tangle we mean a collection of curves (some of them perhaps closed) embedded in $D^{2} \times[0,1]$, with precisely $2 n$ endpoints, $n$ in $D^{2} \times\{0\}$ and $D^{2} \times\{1\}$ each, at the prescribed marked points in the disk. The tangles are framed, ie they are given with a trivialization of their normal bundle. This is necessary since the $q^{ \pm 2(1-N)}$-skewed versions of the first Reidemeister move in the Kauffman relations above are inconsistent with invariance under the first Reidemeister move. As with $\mathrm{TL}_{n}$, the multiplication is given by vertical stacking. Like above, $\operatorname{BMW}(N)=\bigcup_{n} \operatorname{BMW}(N)_{n}$.

The Markov trace $\operatorname{tr}_{K}: \operatorname{BMW}(N)_{n} \longrightarrow \mathbb{Z}\left[q, q^{-1}\right]$ is defined on the generators by connecting the top and bottom endpoints by standard parallel arcs in the complement of $D^{2} \times[0,1]$ in 3-space, sweeping from top to bottom, and computing the $\mathrm{SO}(N)$ Kauffman polynomial (using the above skein relations) of the resulting link. Below we will discuss this trace in detail.

Since the object of main interest in this paper is the $\mathrm{SO}(3)$ algebra, we will omit $N=3$ from the notation, and set $\mathrm{BMW}_{n}=\operatorname{BMW}(3)_{n}$.

\subsection{The chromatic polynomial and the chromatic algebra}

The chromatic polynomial $\chi_{\Gamma}(Q)$ of a graph $\Gamma$, for $Q \in \mathbb{Z}_{+}$, is the number of colorings of the vertices of $\Gamma$ with the colors $1, \ldots, Q$ where no two adjacent vertices have the same color. To study $\chi_{\Gamma}(Q)$ for noninteger values of $Q$, it is convenient to use the contraction-deletion relation. Given any edge $e$ of $\Gamma$ which is not a loop,

$$
\chi_{\Gamma}(Q)=\chi_{\Gamma \backslash e}(Q)-\chi_{\Gamma / e}(Q),
$$

where $\Gamma \backslash e$ is the graph obtained from $\Gamma$ by deleting $e$, and $\Gamma / e$ is obtained from $\Gamma$ by contracting $e$. (If $\Gamma$ contains a loop then $\chi_{\Gamma} \equiv 0$ ). Note: while discussing the chromatic algebra, we will interchangeably use two variables, $Q$ and $q$, where $Q=\left(q+q^{-1}\right)^{2}$. 
The defining contraction-deletion rule (2-1) may be viewed as a linear relation between the graphs $G, G / e$ and $G \backslash e$, so in this context it is natural to consider the vector space defined by graphs, rather than just the set of graphs. Thus consider the set $\mathcal{G}_{n}$ of the isotopy classes of planar graphs $G$ embedded in a rectangle with $n$ endpoints at the top and $n$ endpoints at the bottom of the rectangle, and let $\mathcal{F}_{n}$ denote the free algebra over $\mathbb{Z}\left[q, q^{-1}\right]$ with free additive generators given by the elements of $\mathcal{G}_{n}$. As usual, the multiplication is given by vertical stacking in the plane.

The local relations among the elements of $\mathcal{G}_{n}$, analogous to contraction-deletion rule for the chromatic polynomial, are given in the figures below. To be precise, the chromatic algebra relations are dual to the relations defining the chromatic polynomial, the reason for this choice is to make the connection with the TL and BMW algebras more natural; see Section 2.6 below. Note that these relations only apply to inner edges which do not connect to the top and the bottom of the rectangle. They are

If $e$ is an inner edge of a graph $G$ which is not a loop, then $G=G / e-G \backslash e$.

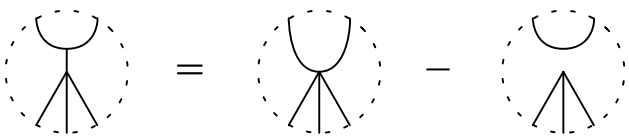

If $G$ contains an inner edge $e$ which is a loop, then $G=\left(q^{2}+1+q^{-2}\right) G \backslash e$. (In particular, this relation applies if $e$ is a simple closed curve not connected to the rest of the graph.) If $G$ contains a $1-$ valent vertex (in the interior of the rectangle) then $G=0$.

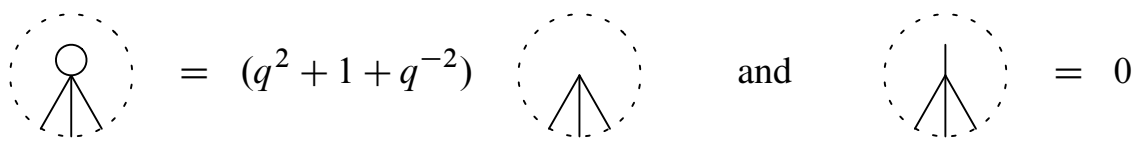

Again, note that the relations (2-2), (2-3) are dual to those defining the chromatic polynomial. In particular, the relation on the left in (2-3) amounts to removing a univalent vertex in the dual graph, giving rise to the factor $\left(q^{2}+1+q^{-2}\right)$. The relation on the right in (2-3) holds since the dual graph contains a loop.

2.5 Definition (Fendley-Krushkal [5]) The chromatic algebra in degree $n, \mathcal{C}_{n}$, is an algebra over $\mathbb{Z}[q]$ which is defined as the quotient of the free graph algebra $\mathcal{F}_{n}$ by the ideal $I_{n}$ generated by the relations (2-2), (2-3) above. Set $\mathcal{C}=\bigcup_{n} \mathcal{C}_{n}$.

The trace, $\operatorname{tr}_{\chi}: \mathcal{C} \longrightarrow \mathbb{Z}[q]$ is defined on the additive generators (graphs $G$ in the rectangle $R$ ) by connecting the top and bottom endpoints of $G$ by disjoint arcs in 
complement of $R$ the plane (denote the result by $\bar{G}$ ) and evaluating the chromatic polynomial of the dual graph $\widehat{\bar{G}}$ :

$$
\operatorname{tr}_{\chi}(G)=\left(q+q^{-1}\right)^{-2} \cdot \chi_{\hat{\bar{G}}}\left(\left(q+q^{-1}\right)^{2}\right) .
$$

\subsection{Relations between the diagrammatic algebras}

This section recalls trace-preserving homomorphisms between the $\mathrm{SO}(3) \mathrm{BMW}$, chromatic, and Temperley-Lieb algebras. A categorified version is given in Sections 5 and 6 below.

2.7 Definition The formulas (introduced by Kauffman and Vogel [10])

$$
\begin{aligned}
& \backslash \mapsto q^{-2}||-X+q^{2} \bigcap \\
& 久 \mapsto q^{2} \mid-X+q^{-2} \bigcup
\end{aligned}
$$

define a homomorphism of algebras $i: \mathrm{BMW}_{n} \longrightarrow \mathcal{C}_{n}$ over $\mathbb{Z}\left[q, q^{-1}\right]$; see Fendley and Krushkal [5, Theorem 5.1] (see also Fendley and Read [6]).

2.8 Definition Define a homomorphism $\phi: \mathcal{F}_{n} \longrightarrow \mathrm{TL}_{2 n}$ on the additive generators (graphs in a rectangle) of the free graph algebra $\mathcal{F}_{n}$ by replacing each edge with the second Jones-Wenzl projector $P_{2}$, and resolving each vertex as shown in the figure below:

$$
\mapsto \underset{\uparrow}{\downarrow}=||-\frac{1}{q+q^{-1}} \bigcup_{1} \text { and } \searrow \mapsto\left(q+q^{-1}\right)
$$

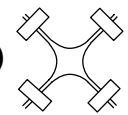

The factor in the definition of $\phi$ corresponding to an $r$-valent vertex is $\left(q+q^{-1}\right)^{(r-2) / 2}$, so for example it equals $q+q^{-1}$ for the 4 -valent vertex in the figure above. The overall factor for a graph $G$ is the product of the factors $\left(q+q^{-1}\right)^{(r(V)-2) / 2}$ over all vertices $V$ of $G$.

Therefore $\phi(G)$ is a sum of $2^{E(G)}$ terms, where $E(G)$ is the number of edges of $G$. It is shown in Lemmas 6.2 and 6.4 in [5] that $\phi$ induces a well-defined homomorphism of algebras $\mathcal{C}_{n} \longrightarrow \mathrm{TL}_{2 n}$. Moreover,

$$
\operatorname{tr}_{\chi}(G)=\operatorname{tr}_{\mathrm{TL}}(\phi(G)) .
$$


Phrased differently, up to a renormalization factor $\left(q+q^{-1}\right)^{-2}$ the chromatic polynomial of a planar graph may be computed as the Yamada polynomial [17] of the dual graph, that is the evaluation of the quantum spin network where each edge is labeled with the second projector. The following lemma summarizes the above discussion:

2.9 Lemma The homomorphisms $i, \phi$ are trace-preserving, in other words the following diagram commutes:

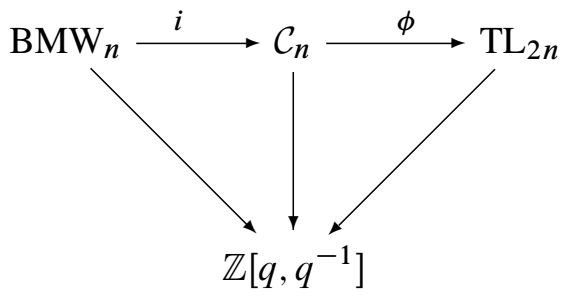

We refer the reader to Fendley and Krushkal [5] for a more detailed discussion of the properties and relations between the $\mathrm{SO}(3) \mathrm{BMW}$, chromatic, and Temperley-Lieb algebras.

\section{Categorification of the Temperley-Lieb algebra}

In this section we recall Dror Bar-Natan's graphical formulation [1] of Khovanov's categorification of the Temperley-Lieb algebra [11].

There is an additive category $\operatorname{Pre}-\operatorname{Cob}(n)$ whose objects are isotopy classes of formally $q$-graded Temperley-Lieb diagrams with $2 n$ boundary points. The morphisms are given by the free $\mathbb{Z}$-module spanned by isotopy classes of orientable cobordisms bounded in $\mathbb{R}^{3}$ between two planes containing such diagrams. If $\chi(S)$ is the Euler characteristic of a surface $S$, then a cobordism $C: q^{i} A \rightarrow q^{j} B$ has degree given by

$$
|C|=\chi(C)-n+j-i .
$$

It has become a common notational shorthand to represent a handle by a dot and a saddle by a flattened diagram containing a dark line:

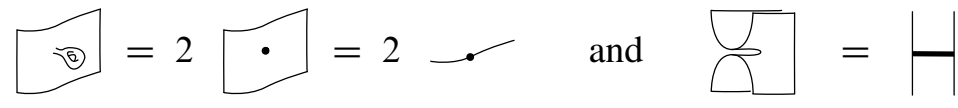


There are maps from a circle to the empty set and vice versa given by a punctured sphere and a punctured torus:

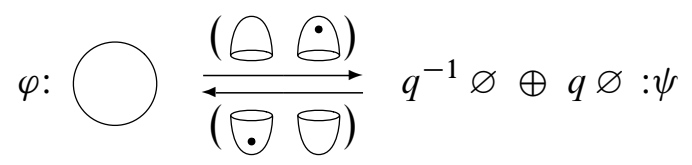

In order to obtain $\varphi \circ \psi=1$ and $\psi \circ \varphi=1$ we form a new category $\operatorname{Cob}(n)=\operatorname{Cob}_{\cdot / l}^{3}(n)$ obtained as a quotient of the category $\operatorname{Pre}-\operatorname{Cob}(n)$ by the relations given below.

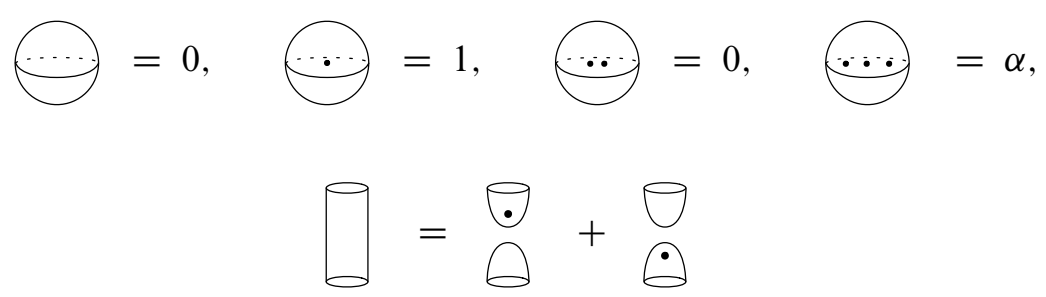

Notice that a sheet with two dots is equal to $\alpha$ times a sheet with no dots, as a consequence of the neck-cutting relation and the definition of $\alpha$ above. The cylinder or neck cutting relation implies that closed surfaces $\Sigma_{g}$ of genus $g>3$ must evaluate to 0 . In what follows we will let $\alpha$ be a free variable and absorb it into our base ring. One can think of $\alpha$ as a deformation parameter.

In this categorification the skein relation becomes

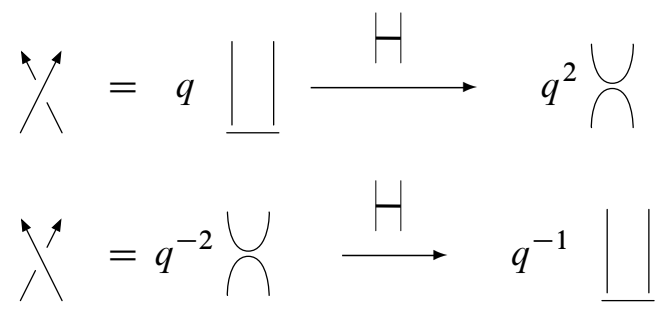

where the underlined diagram represents homological degree 0 .

3.1 Definition Let $\operatorname{Kom}(n)=\operatorname{Kom}\left(\operatorname{Mat}\left(\operatorname{Cob}_{\cdot}^{3} / l(n)\right)\right)$ be the category of chain complexes of formal direct sums of objects in $\mathrm{Cob}_{\cdot}^{3} / l(n)$.

The skein relation allows us to associate to any tangle diagram $D$ with $2 n$ boundary points an object in $\operatorname{Kom}(n)$.

Given two objects $C, D \in \operatorname{Kom}(n)$ we will use $C \otimes D$ to denote the categorified Temperley-Lieb multiplication $\otimes: \operatorname{Kom}(n) \otimes \operatorname{Kom}(n) \rightarrow \operatorname{Kom}(n)$ obtained by gluing 
all diagrams and morphisms along the $n$ boundary points and $n$ boundary intervals respectively.

\subsection{Chain homotopy lemmas}

We will make frequent use of the following standard lemma:

3.3 Lemma (Gaussian elimination [2]) Let $K_{*}$ be a chain complex in an additive category $\mathcal{A}$ containing a summand of the form given below:

$$
A \stackrel{(\dot{\delta})}{\longrightarrow} B \oplus C \stackrel{\left(\begin{array}{ll}
\varphi & \lambda \\
\mu & \eta
\end{array}\right)}{\longrightarrow} D \oplus E \stackrel{(\cdot \epsilon)}{\longrightarrow} F
$$

Then if $\varphi: B \rightarrow D$ is an isomorphism there is a homotopy equivalence from $K_{*}$ to a smaller complex containing the summand below obtained by removing $B$ and $D$ terms via $\varphi$ :

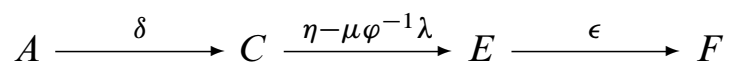

The following result is a direct generalization which will be very useful in our context.

3.4 Lemma (Simultaneous Gaussian elimination [4]) Let $K_{*}$ be a chain complex in an additive category $\mathcal{A}$ of the form

$$
K_{*}=A_{0} \oplus C_{0} \stackrel{M_{0}}{\longrightarrow} A_{1} \oplus B_{1} \oplus C_{1} \stackrel{M_{1}}{\longrightarrow} A_{2} \oplus B_{2} \oplus C_{2} \stackrel{M_{2}}{\longrightarrow} \cdots
$$

where $\quad M_{0}=\left(\begin{array}{cc}a_{0} & c_{0} \\ d_{0} & f_{0} \\ g_{0} & j_{0}\end{array}\right) \quad$ and $\quad M_{i}=\left(\begin{array}{ccc}a_{i} & b_{i} & c_{i} \\ d_{i} & e_{i} & f_{i} \\ g_{i} & h_{i} & j_{i}\end{array}\right) \quad$ for all $i>0$.

If $a_{2 i}: A_{2 i} \rightarrow A_{2 i+1}$ and $e_{2 i+1}: B_{2 i+1} \rightarrow B_{2 i+2}$ are isomorphisms for $i \geq 0$ then the chain complex $K_{*}$ is homotopy equivalent to the smaller chain complex $D_{*}$ obtained by removing all $A_{i}$ and $B_{i}$ terms via the isomorphisms $a_{2 i}$ and $e_{2 i+1}$ :

$$
D_{*}=C_{0} \stackrel{q_{0}}{\longrightarrow} C_{1} \stackrel{q_{1}}{\longrightarrow} C_{2} \stackrel{q_{2}}{\longrightarrow} C_{3} \stackrel{q_{3}}{\longrightarrow} \cdots
$$

where $q_{2 i}=j_{2 i}-g_{2 i} a_{2 i}^{-1} c_{2 i}$ and $q_{2 i+1}=j_{2 i+1}-h_{2 i+1} e_{2 i+1}^{-1} f_{2 i+1}$.

\section{Construction of the second projector}

In this section we define a chain complex $P_{2} \in \operatorname{Kom}(2)$ which categorifies the second Jones-Wenzl projector (Definition 2.2). This construction of $P_{2}$ is universal and unique up to homotopy by Cooper and Krushkal [4]. (Other definitions were obtained by Frenkel, Stroppel and Sussan [7] and Rozansky [14]). 


\subsection{The second projector revisited}

The second projector 兑 is defined to be the chain complex
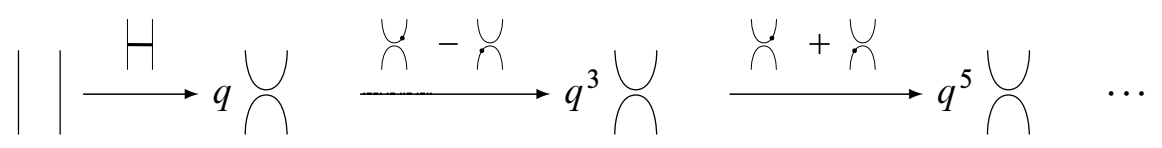

in which the last two maps alternate ad infinitum. More explicitly,

$$
P_{2}=\left(C_{*}, d_{*}\right),
$$

the chain groups are given by

$$
C_{n}= \begin{cases}q^{0}|| & n=0, \\ q^{2 n-1} \bigcup & n>0,\end{cases}
$$

and the differential is given by

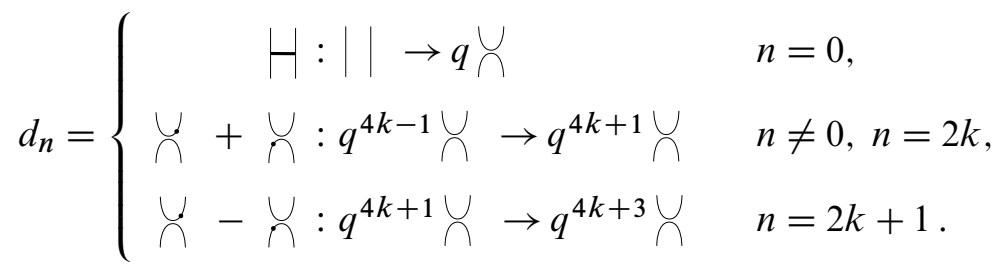

4.2 Proposition $P_{2}$ defined above is a chain complex.

Proof Since $d_{2 n+1} \circ d_{2 n}=d_{2 n} \circ d_{2 n-1}$ there are only two cases:

$$
\begin{aligned}
& d_{1} \circ d_{0}=H-H \\
& =H-H=0 \text {, } \\
& d_{2 n+1} \circ d_{2 n}=\left(\breve{\Upsilon}+\bigcup_{n}\right) \circ\left(\breve{\Upsilon}-\bigcup_{n}\right)
\end{aligned}
$$

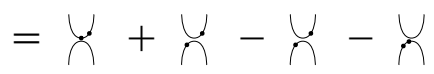

$$
\begin{aligned}
& =\alpha \bigvee+0-\alpha \bigvee=0 \text {. }
\end{aligned}
$$


4.3 Theorem [4] The chain complex $P_{2} \in \operatorname{Kom}(2)$ defined above is contractible "under turnback" and a homotopy idempotent. Graphically,
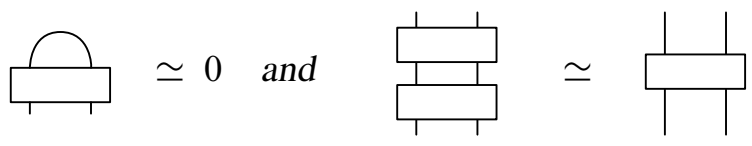

Algebraically, these are the relations

$$
P_{2} \otimes e_{1} \simeq 0 \simeq e_{1} \otimes P_{2} \quad \text { and } \quad P_{2} \otimes P_{2} \simeq P_{2} .
$$

Proof We will prove the turnback property first. Note that the vertical symmetry in the definition of $P_{2}$ implies $P_{2} \otimes e_{1} \cong e_{1} \otimes P_{2}$. Consider $e_{1} \otimes P_{2}$ :

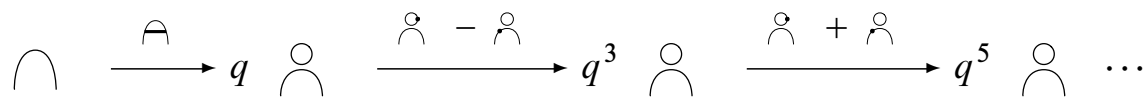

We "deloop" and conjugate our differentials by the isomorphism $\varphi$ in Section 3 to obtain the isomorphic complex

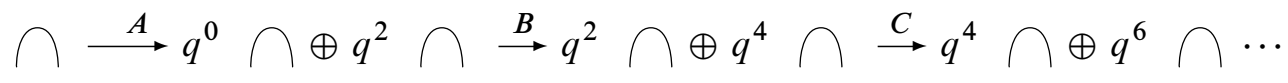

where $\quad A=\left(\begin{array}{l}\cap \\ \cap\end{array}\right), \quad B=\left(\begin{array}{cc}-\cap & \cap \\ \alpha \cap & -\cap\end{array}\right), \quad C=\left(\begin{array}{cc}\cap & \cap \\ \alpha \cap & \cap\end{array}\right)$.

Applying Lemma 3.4 (simultaneous Gaussian elimination) by using the identity map in the first component of the first map and the identity in the upper righthand component of each successive matrix shows that the complex is homotopic to the zero complex.

The relation $P_{2} \otimes P_{2} \simeq P_{2}$ follows from expanding either the top or bottom projector and again using Lemma 3.4 to contract all of the projectors containing turnbacks as above. What remains is the chain complex for $P_{2}$ in degree 0 .

\section{Categorification of the $\mathrm{SO}(3) \mathrm{BMW}$ algebra}

In this section we show that the chain complexes obtained by applying the second projector to the strands of a 2-cabling are invariant under Reidemeister moves and satisfy relations categorifying those of the $\mathrm{SO}(3)$ BMW algebra.

Given a diagram $D$, replace each strand in $D$ with two parallel strands composed with the second projector. Denote the resulting total complex by $F(D)$ in the category 
$\operatorname{Kom}(2 n)$. (Note that using the categorified Kauffman skein relation in Section 3 one associates a chain complex to oriented tangles and the two parallel strands in the current construction are given opposite orientations). This can be illustrated by:

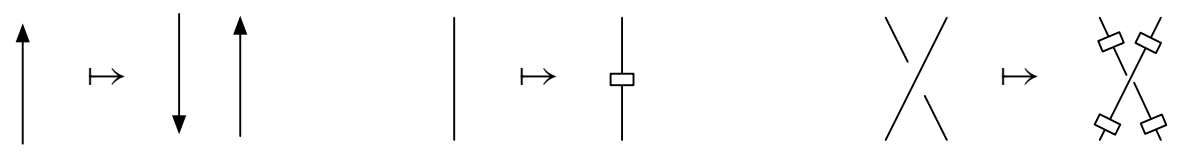

Formally, this construction categorifies the 2-colored Jones polynomial; see [4] and Section 8.4 for further discussion. In the remainder of this section we prove that the Reidemeister moves and $\mathrm{SO}(3)$ skein relation are satisfied up to homotopy.

5.1 Lemma (Projector isotopy) A free strand can be moved over or under a projector up to homotopy. In pictures:

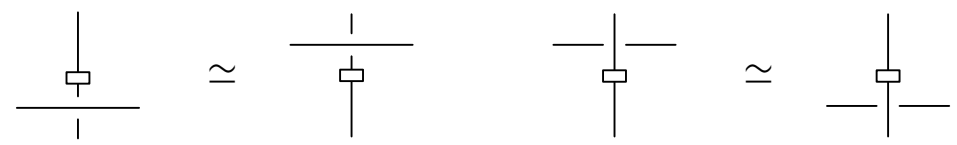

Proof The chain complex for the diagram with the projector below the strand and the chain complex for the diagram with the projector above the strand are chain homotopy equivalent to the chain complex $C$ for the diagram with two projectors: one above the strand and one below the strand. This is true because expanding either of the two projectors in $C$ gives the identity diagram in degree zero and every other term involves a turnback, which is contractible when combined with the second copy of the projector.

This lemma allows us to show that the Reidemeister moves are satisfied.

5.2 Theorem This construction yields invariants of framed tangles.

Proof For the second Reidemeister move:

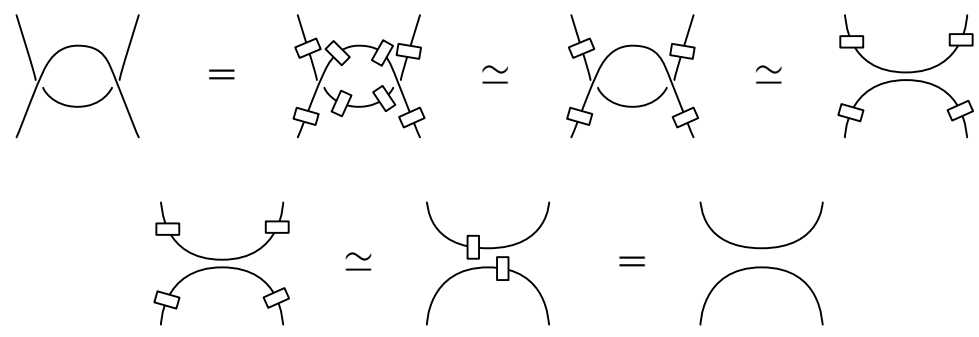


The first equality is by definition. The homotopy equivalence follows from the projector isotopy lemma and $P_{2} \otimes P_{2} \simeq P_{2}$. We then apply the original second Reidemeister move and $P_{2} \otimes P_{2} \simeq P_{2}$ again. The argument for the third Reidemeister move features the same ideas:
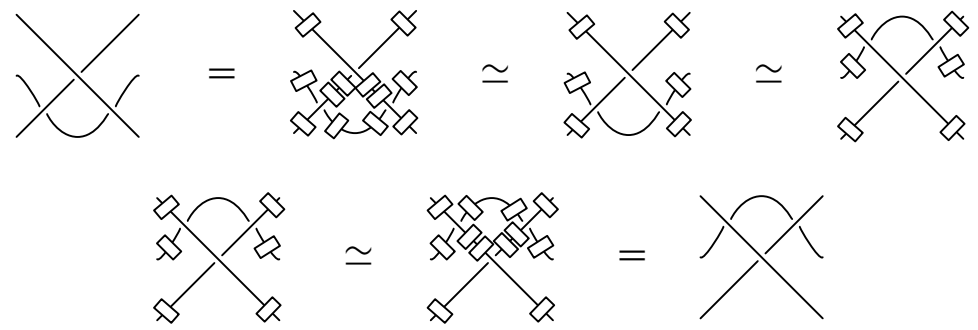

The $q^{ \pm 4}$-skewed version of first Reidemeister move (Section 2.3) are satisfied by our construction.

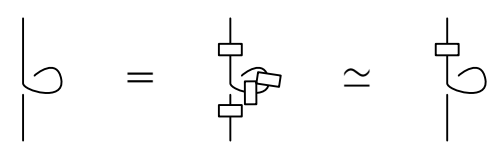

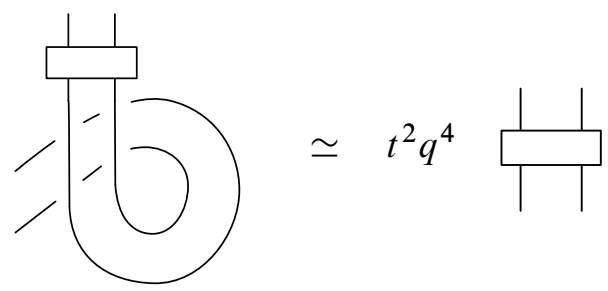

where $t^{2} q^{4}$ denotes bidegree $(2,4)$. This is obtained by expanding all of the crossings, delooping and contracting the remaining subcomplex consisting of projectors containing turnbacks. We've shown

$$
\bigcap \simeq q^{2(N-1)}
$$

with $N=3$. The opposite crossing follows from the same argument.

\subsection{SO(3) BMW skein relation}

In order to prove that the first skein relation pictured in Section 2.3 is satisfied by our categorification we consider the chain complex associated to a crossing:

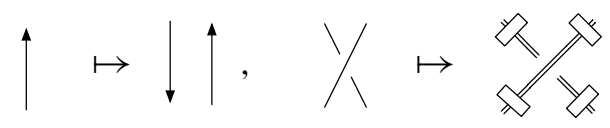


Now expanding all four crossings on the right hand side yields a chain complex with 16 terms. (The reader may find it helpful to draw the diagram with all 16 terms to follow the argument below.) We will use the convention below to index resolutions:

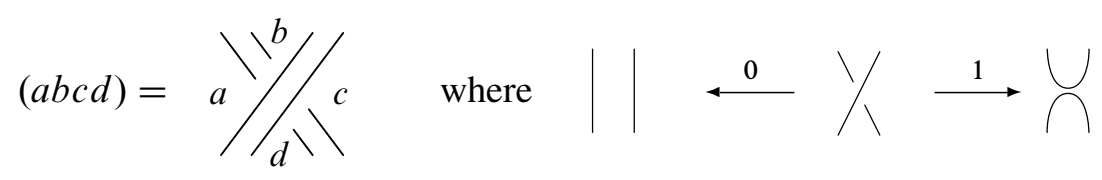

There is one circle corresponding to the (0101) resolution which can be delooped and Gaussian elimination can be performed removing the terms corresponding to the (0001) resolution and the (1101) resolution. Nine of the remaining terms contain projectors with turnbacks. ${ }^{1}$ Contracting using Lemma 3.4 these yields the chain complex

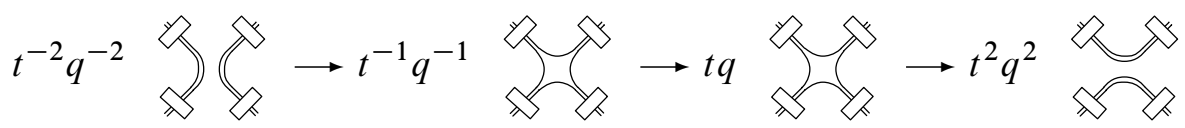

giving a categorification of the crossing formula in Definition 2.7. The factor $\left(q+q^{-1}\right)$ which comes from the two terms in the middle is seen in the translation of the 4-valent graph to the Temperley-Lieb algebra (see Definition 2.8 of the homomorphism $\phi$.) Note that the diagram above is only a schematic illustration of the chain complex for the resolution of the crossing at the beginning of Section 5.3: the contractions mentioned above produce maps which are not illustrated in the above diagram. Next we will examine this chain complex in more detail.

We now proceed to show that the relation

$$
Y-\searrow^{\prime}=\left(q^{2}-q^{-2}\right)(\mid-\bigcup)
$$

holds in our category, this requires a more detailed analysis of the chain complex considered above. Begin by again expanding all four crossings in (5-1), corresponding to the leftmost term in the equation above. We obtain a chain complex with 16 terms with one term in homological degrees -2 and 2, four terms in degrees -1 and 1 and

\footnotetext{
${ }^{1}$ Those corresponding to (1000), (0010), (1100), (1010), (1001), (0110), (0011), (1110) and (1011) resolutions.
} 
six terms in degree 0 . Form a new chain complex:

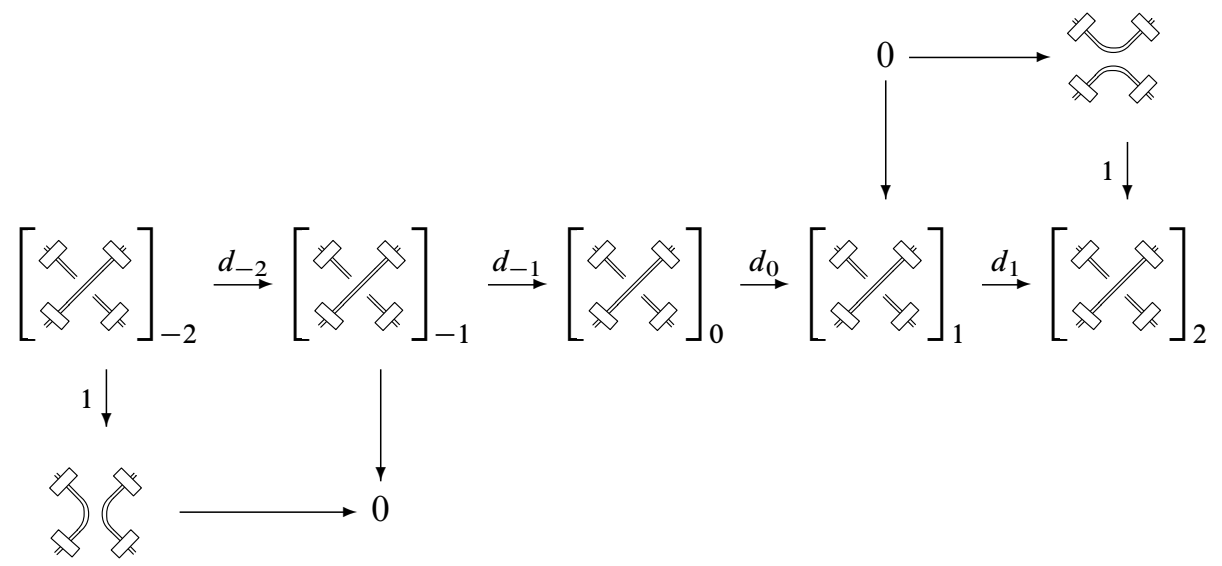

The graded Euler characteristic of this complex is the quadrivalent vertex in Definition 2.8. Contracting the first and last maps using the introduced isomorphisms yields the chain complex:

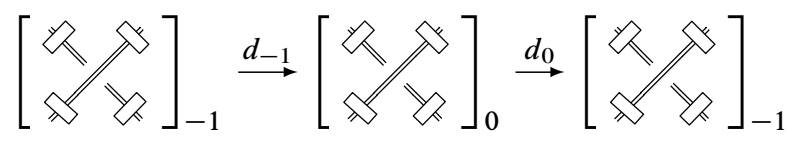

The maps $d_{-1}$ and $d_{0}$ remain the same as in the previous diagram and so consist of saddles between resolutions of crossings. Now contract terms in degrees -1 and 1 that are diagrams with projectors capped by turnbacks ${ }^{2}$. Observe again that contracting these will not affect the maps between remaining terms. There remains a contractible term (1010) in degree zero (with four turnbacks) which is a direct summand of the chain complex, that is there are no arrows starting or ending at this term, so that contracting this term does not affect the maps between the remaining terms. Again delooping the term in the center corresponding to the (0101) resolution allows one to cancel terms corresponding to (0001) and (0111) resolutions in degrees -1 and 1 respectively. These cancelations in fact do change the maps between the remaining terms, the resulting maps can be analyzed using the Gaussian elimination Lemma 3.3, and the result is given below. The chain complex

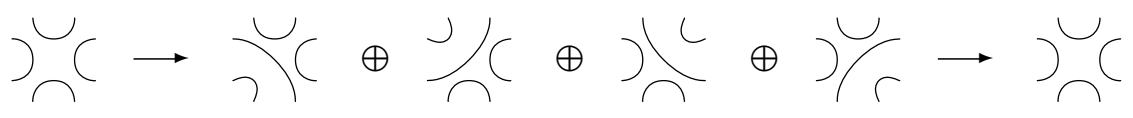

${ }^{2}$ Terms corresponding to (1000), (0010), (1110) and (1011) resolutions. 
is what remains. All of the maps are saddles. Note that all of the diagrams contain four projectors which are not pictured. The first and last terms are the chain complex associated to the planar crossing (the middle term in the equality below), while the four terms in the middle have a projector capped with a turnback, and are therefore contractible.

On the other hand, expanding the lefthanded crossing in (5-2) rather than the righthanded one and carrying out the same argument yields precisely the same complex! This is clear since the terms in the diagram above are $\pi / 2$ rotationally symmetric. It follows that on the level of the graded Euler characteristic,

$$
q^{2}||-Y+q^{-2} \bigcap=X=q^{-2}||-Y^{\prime}+q^{2} \bigcup
$$

which is equivalent to the desired relation (5-2).

\section{Chromatic categorification}

In this section we show that our construction produces a categorification of the chromatic polynomial of planar graphs. To each planar graph $G$ we associate a chain complex $\langle G\rangle$ whose graded Euler characteristic is a particular normalization of the chromatic polynomial.

Our construction differs in significant ways from other categorifications of the chromatic polynomial present in the literature; see Helme-Guizon and Rong [9] and Stošić [15]. In particular, it depends on a specific choice of Frobenius algebra. This follows from the relations in Section 3. While this rigidity may have the disadvantage of limiting the variety of answers that our theory provides, it allows for an extension to invariants of ribbon graphs embedded in $\mathbb{R}^{3}$. This information then enriches the structure of the underlying chromatic polynomial. See Section 6.4 for more details.

In Section 6.3 below we show that to each edge $e \in G$ which is not a loop there is a contraction-deletion long exact sequence on the homology of $\hat{G}$ corresponding to the contraction-deletion relation of Section 2.4.

\subsection{A categorification of the chromatic polynomial}

In order to associate to a planar graph $G$ a chain complex $\langle G\rangle$ with the correct Euler characteristic, we define $\langle G\rangle$ to be the evaluation of the dual graph $\widehat{G}$ in the $\operatorname{SO}(3)$ 
BMW categorification of Section 5. For example:

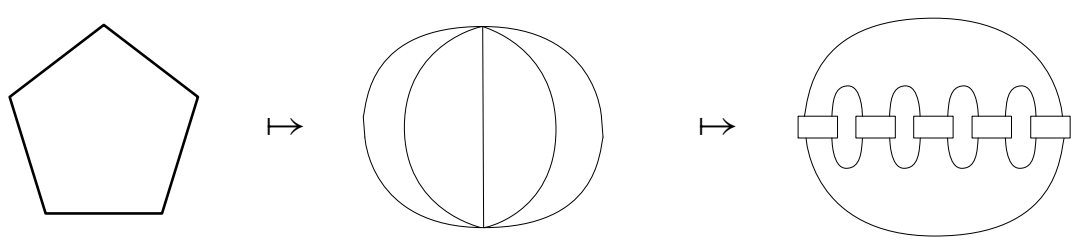

The pentagon is dual to the graph $\theta_{5}$ to its right. Associated to $\theta_{5}$ is the chain complex given in Section 6.4: replace each edge with a pair or parallel strands with the second Jones-Wenzl projector, and connect the strands near each vertex to get a planar diagram. (The homology of $\theta_{n}$ for all $n>1$ is given in the Appendix).

Defining maps between planar graphs $G$ and $H$ to be chain maps between the associated chain complexes $\langle G\rangle$ and $\langle H\rangle$ yields a category $\mathcal{C}^{\prime}$.

6.2 Theorem If $G$ is a planar graph then up to a normalization the graded Euler characteristic of $\langle G\rangle$ is the chromatic polynomial $\chi_{G}$ evaluated at $\left(q+q^{-1}\right)^{2}$ :

$$
\chi_{G}\left(\left(q+q^{-1}\right)^{2}\right)=\left(q+q^{-1}\right)^{2} \prod_{v}\left(q+q^{-1}\right)^{(r(v)-2) / 2} \chi_{q}\langle G\rangle,
$$

where the product is taken over all vertices $v$ of the dual graph $\widehat{G}$ and $r(v)$ is the valence of $v$ (see Definition 2.8). The above equation holds in the ring of formal power series $\mathbb{Z} \llbracket q \rrbracket$.

The proof of this theorem follows immediately from the discussion in Section 2.6 and Lemma 2.9. (To be precise, $\langle G\rangle$ is a categorification of the Yamada polynomial of the dual graph [17] which is defined as the evaluation of the spin network where each edge is labeled with the second projector; this accounts for the difference between the normalization above and that of (2-4) of Section 2.4).

\subsection{The contraction-deletion rule}

The chain complex $\langle G\rangle$ associated to a planar graph $G$ in Section 6.1 above satisfies a version of the contraction-deletion rule. For any edge $e \in G$ which is not a loop there is an exact triangle

$$
[G / e] \longrightarrow\langle G\rangle \longrightarrow\langle G \backslash e\rangle
$$

in the category $\mathcal{C}^{\prime}$. Here $\langle G \backslash e\rangle$ is as in Section 6.1 and $[G / e]$ is the chain complex obtained by removing the first term from the expansion of the projector labelling 
the edge $e$ in the chain complex $\langle G\rangle$ (as illustrated below). Note that $[G / e]$ has graded Euler characteristic $\left(q+q^{-1}\right)^{-1}\langle G / e\rangle$ in the power series ring (compare to Definition 2.2). There is a functor $F$ from the category $\mathcal{C}^{\prime}$ to abelian groups, given by associating to each circle a Frobenius algebra [1]. The homology groups of chain complexes fitting into any exact triangle form a long exact sequence in the image of $F$ [16, 10.1.4, page 372].

Let $e$ be an edge (not a loop) of a planar graph $G$. Consider the edge of the dual graph, intersecting the edge $e$ in a single point. The construction sends this dual edge to two parallel lines with a projector as in the figure on the left in (6-2). By definition (Section 4.1) this projector is expanded into the chain complex

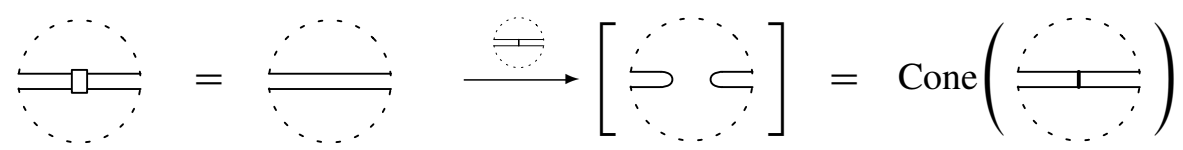

where all of the terms besides the first one have been collected into the chain complex with brackets on the right hand side above. Note that the homological degree zero term of this chain complex is the chain complex associated to $G \backslash e$. Now the cone on the inclusion map $[G / e] \longrightarrow\langle G\rangle$ is chain homotopy equivalent to $\langle G \backslash e\rangle$. This gives an exact triangle by definition of the Cone complex [16, pages 18, 371]. Dualizing again yields the exact triangle (6-1).

Note that on the level of the graded Euler characteristic (6-1) corresponds to a renormalized version of the contraction-deletion rule: the term $\chi_{\Gamma / e}$ in (2-1) acquires a coefficient $\left(q+q^{-1}\right)^{-1}$. This version of the contraction-deletion rule corresponds to the renormalized chromatic polynomial discussed in Theorem 6.2.

The other two defining relations (2-3) of the chromatic algebra also have analogues at the level of chain complexes. The second relation in (2-3) is an immediate consequence of Theorem 4.3 since a univalent vertex gives rise to a projector capped with a turnback. The interested reader is encouraged to derive the chain complex analogue of the (renormalized, as discussed above) first relation in (2-3). This involves expanding the projector labeling the loop and then performing a delooping and Gaussian elimination, analogous to a step in the calculation of the trace of the second projector in Section 7.1 below.

\subsection{Ribbon graphs}

A ribbon graph is a pair $(G, S)$ where $G$ is a graph embedded in a surface $S$ with boundary, and the inclusion $G \subset S$ is a homotopy equivalence. Our construction gives 
an invariant of ribbon graphs embedded in the 3-sphere. Specifically, to a ribbon graph $(G, S)$ associate a chain complex as follows: Replace each edge of $G$ with the second Jones-Wenzl projector $P_{2}$, and using the ribbon structure resolve each vertex as in the figure below:

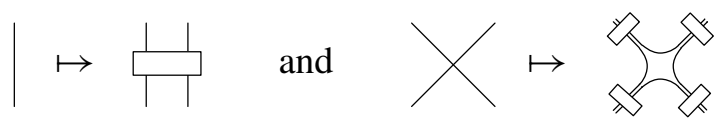

The resulting curves in the neighborhood of each vertex are oriented as the boundary of a regular neighborhood of the graph $G$ in $S$.

It is an interesting question to determine how powerful this invariant is, and in particular whether this homology theory may be used to detect planar graphs. Given a connected ribbon graph $(G, S)$ embedded in $S^{3}$, contracting a maximal tree gives a map to the graph $G^{\prime}$ with a single vertex and a number of loops (with the same underlying surface, embedded in $S^{3}$ ). There is an induced map on chain complexes (which amounts to the projection onto the homological degree zero for each contracted edge; see Section 6.3 below.) If the embedding of $(G, S)$ into $S^{3}$ is isotopic to a planar embedding, then the homology of $G^{\prime}$ is the chromatic homology of a tree, computed in the Appendix. Analyzing the homology of planar graphs motivated the following conjecture.

Conjecture A ribbon graph $(G, S)$ embedded into $S^{3}$ is isotopic to a planar graph if and only if its homology groups $H_{i}$ are trivial for $i<0$, and $H_{0}$ is free of rank 2 .

A related question is to determine whether the genus of the ribbon graph (defined as the genus of the underlying surface $S$ ) is determined by this homology theory.

\section{Computations}

\subsection{Homology of the unknot}

The chain complex associated to the unknot is the "Markov trace" of the second projector $P_{2}$ (Section 4.1). The trace of the second projector $p_{2} \in \mathrm{TL}_{2}$ is given by

$$
\bigcirc=[3]=q^{-2}+1+q^{2} \text {. }
$$


Our categorification has this graded Euler characteristic when the parameter $\alpha=0$. It is however not true that the homology of $\operatorname{tr}\left(P_{2}\right)$ is spanned only by classes that correspond to coefficients of the graded Euler characteristic; the homology contains infinitely many terms which cancel in the graded Euler characteristic. For further discussion, see [4].

Taking the trace of our projector yields a complex with alternating differential:

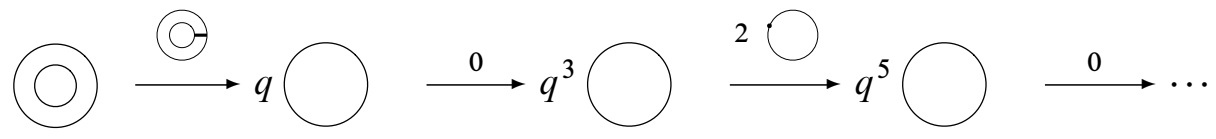

Recall that $8 \alpha=\Sigma_{3}$. The homology of this complex is given by

$$
H_{n}\left(\operatorname{tr}\left(P_{2}\right)\right)= \begin{cases}q^{-2} \mathbb{Z} \oplus q^{0} \mathbb{Z} & n=0, \alpha=0 \text { or } \alpha \neq 0, \\ 0 & n=1, \alpha=0 \text { or } \alpha \neq 0, \\ q^{4 k-2} \mathbb{Z} & n=2 k, \alpha=0, \\ q^{4 k+2} \mathbb{Z} \oplus q^{4 k} \mathbb{Z} / 2 & n=2 k+1, \alpha=0, \\ 0 & n=2 k, \alpha \neq 0, \\ q^{4 k+2} \mathbb{Z} /(2 \alpha) \oplus q^{4 k} \mathbb{Z} / 2 & n=2 k+1, \alpha \neq 0 .\end{cases}
$$

\subsection{Homology of the theta graph}

Consider the theta graph:

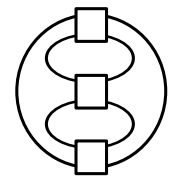

We begin by expanding the middle projector:

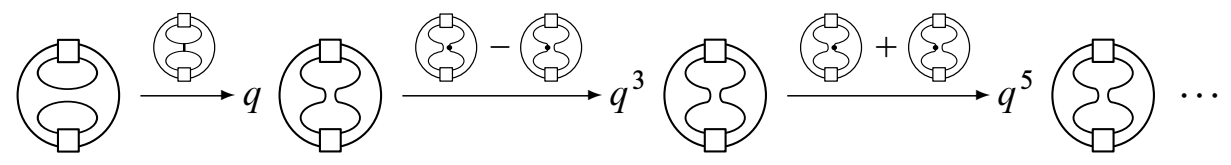


If we then expand the top projector:
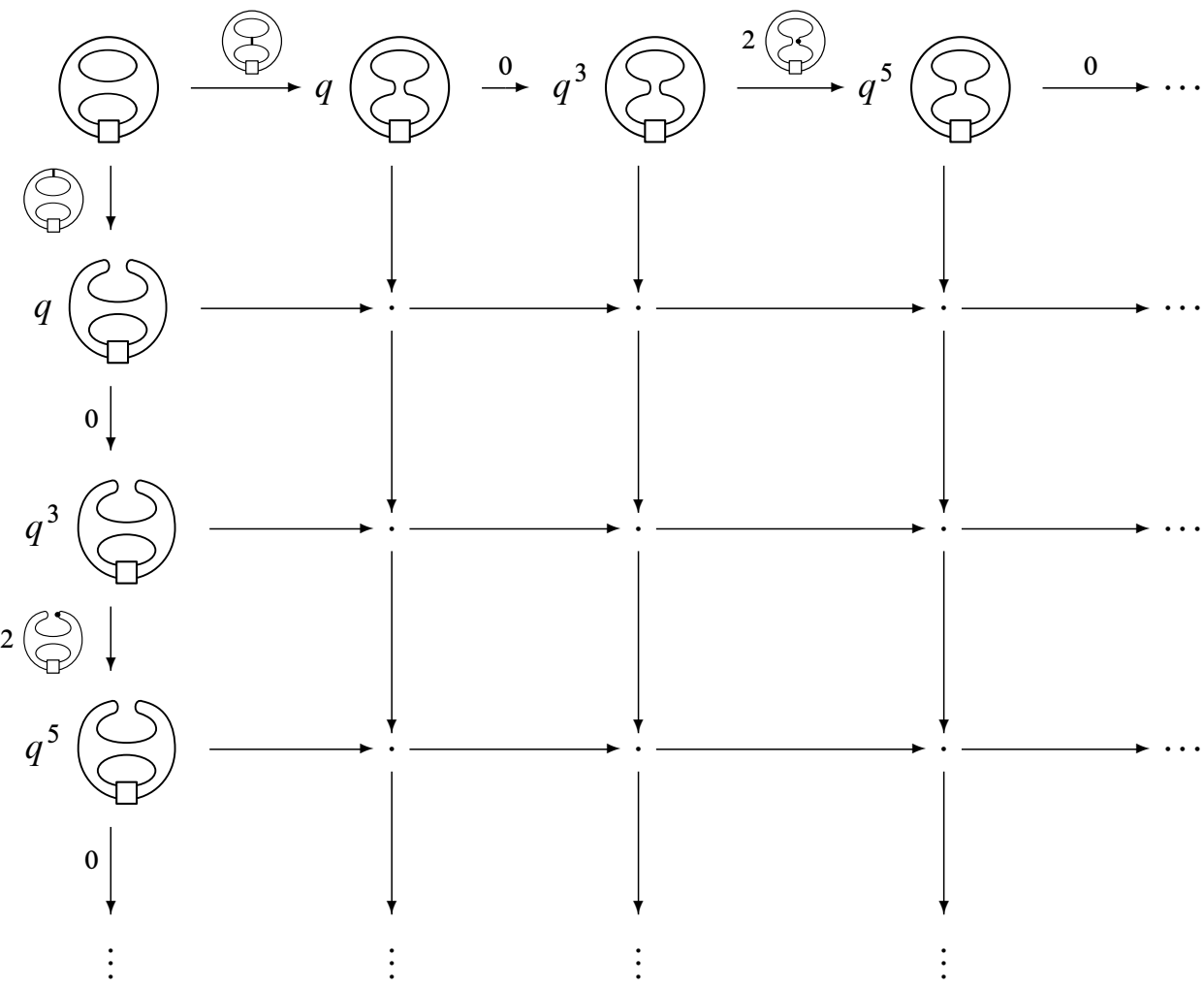

The middle terms are all projectors containing turnbacks, which form a contractible subcomplex. Contracting these yields a homotopy equivalent complex which is a direct sum of the following three complexes:

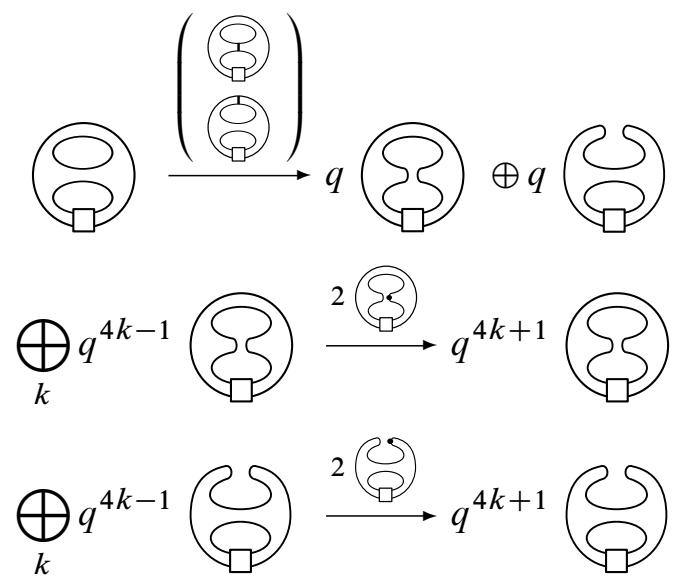


Expanding the projector in each of the bottom two cases shows that these are isomorphic chain complexes. Let's define $E$ to be this chain complex.

In (7-1) the circle can be delooped yielding

$$
q^{-1} E=q^{-1} \bigcirc \rightarrow q \bigcirc
$$

after a Gaussian elimination. We are left with the task of computing $E$. We have:

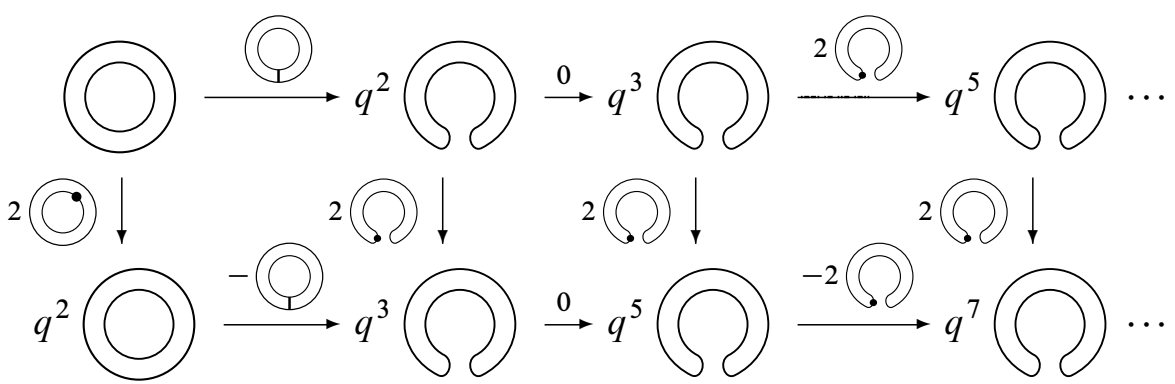

The second column can be removed by delooping leaving a sum of chain complexes of the following form:
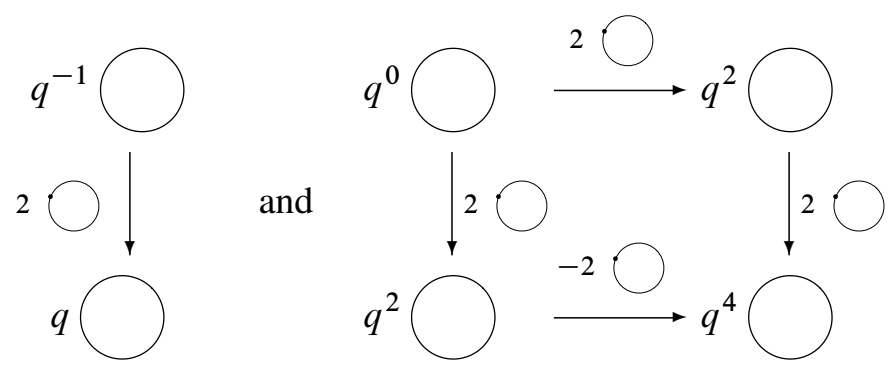

The first complex appears once at the origin of $E$, it has homology $q^{-2} \mathbb{Z}$ in degree 0 and $q^{0} \mathbb{Z} / 2 \oplus q^{2} \mathbb{Z}$ in degree 1 when $\alpha=0$. The second appears countably many times, it has homology $q^{-1} \mathbb{Z}$ in degree $0, q \mathbb{Z} \oplus q \mathbb{Z} / 2 \oplus q^{3} \mathbb{Z}$ in degree 1 and $q^{3} \mathbb{Z} / 2 \oplus q^{5} \mathbb{Z}$ in degree 2. This can be summarized as follows:

$$
\begin{aligned}
& E_{0}=q^{-2} \mathbb{Z} \\
& E_{1}=q^{0} \mathbb{Z} / 2 \oplus q^{2} \mathbb{Z} \\
& E_{2}=q^{2} \mathbb{Z} \\
& E_{3}=q^{4} \mathbb{Z} \oplus q^{4} \mathbb{Z} / 2 \oplus q^{6} \mathbb{Z} \\
& E_{4}=q^{6} \mathbb{Z} \oplus q^{6} \mathbb{Z} / 2 \oplus q^{8} \mathbb{Z} \\
& E_{n}=q^{4} E_{n-2} \text { for } n \geq 5 .
\end{aligned}
$$


If we define $E_{n}=0$ for negative $n$ then we see that, when $\alpha=0$,

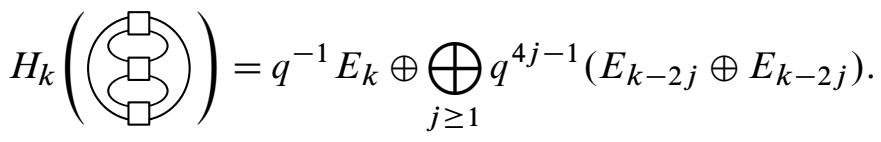

Alternatively, we can write

$$
H(E)=\left(q^{-2}+t q^{2}+t^{2} q^{2}+\frac{t^{3}\left(q^{4}+q^{6}\right)}{1-t q^{2}}\right) \cdot \mathbb{Z} \oplus\left(t+\frac{t^{3} q^{4}}{1-t q^{2}}\right) \cdot \mathbb{Z} / 2
$$

so that $H\left(\theta_{3}\right)=\left(q^{-1}+\frac{2 t^{2} q^{3}}{1-q^{4} t^{2}}\right) \cdot H(E)$.

The Poincaré series for several families of graphs are provided in the Appendix.

\section{Structural observations}

This section states a number of results on the structure of the chromatic homology of planar graphs and of the homology of links. We begin in Section 8.1 with the analysis of the chain maps from the second projector to itself. Section 8.3 states a conjecture on the structure of the chromatic homology of an arbitrary planar graph. In Section 8.4 the homology of knots is shown to split into an interesting "unstable" part, closely related to Khovanov's categorification of the 2-colored Jones polynomial, and a periodic "stable" portion.

\subsection{Homology of the sheet algebra}

Here we start by analyzing maps between objects in Sections 5 and 6 (ie chain complexes associated to 2-colored links and spin networks). Since the categories are built up from local pictures, the first interesting example is given by maps between two intervals. The sheet algebra is defined to be the chain complex of chain maps from the second projector to itself:

$$
\operatorname{End}^{*}(\text { 只 })=\bigoplus_{n} \operatorname{Hom}_{\mathrm{Kom}(2)}\left(\text { 只, } t^{n} \text { 只 }\right) .
$$

This forms a differential graded algebra with differential given by

$$
d_{\text {号 }}(f)=[d, f]=d \circ f+(-1)^{|f|} f \circ d,
$$

ie the graded commutator. The homology of the sheet algebra is given by the homotopy classes of maps from the projector to itself. 
8.2 Theorem The homology of the sheet algebra with $\mathbb{Z}$ coefficients and $\alpha=0$ is given by

$$
H\left(\operatorname{End}^{*}(\text { 兑 })\right)=\mathbb{Z}[u] \oplus \mathbb{Z}[u] \cdot w \oplus \mathbb{Z}[u] /(2 u) \cdot b,
$$

as a $\mathbb{Z}[u]$-module. The algebra multiplication is commutative and determined by $w \cdot b=b^{2}=w^{2}=0$. Representatives for the classes $b, u$, and $w$ are given by the chain maps

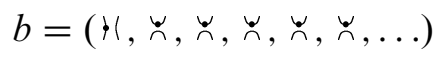

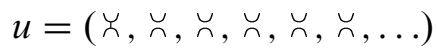

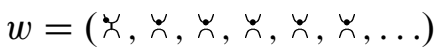

respectively. These have homological degree

$$
\operatorname{deg}(b)=0, \quad \operatorname{deg}(u)=-2 \quad \text { and } \quad \operatorname{deg}(w)=-3 .
$$

The proof is by direct computation. A conceptual explanation is provided by noting that both the trace of 兑 (Section 7.1) and End* (兑) compute the Hochschild homology of the Khovanov ring $H^{1}$ [11]. Note that $b$ is the class of the "dotted identity" 兑. The maps 兑, 兑, and 总 are also chain maps, but they are all homotopic to \pm 兑:

$$
\text { 兑 } \simeq \text { 并 } \simeq \text { 一兑 } \simeq \text { 一觉. }
$$

The homology of the sheet algebra is finite dimensional as a module over the subalgebra generated by $u$. The map $u$ shifts all of the homology down by two degrees. As a chain map, all of its components are isomorphisms except the first which is a saddle. The kernel of the map induced by $u$ may be thought of as "unstable" homology in low degree. The rest of the homology associated to a graph or knot could be called "stable." It is useful to keep these concepts in mind when interpreting the computations contained in this paper.

There is an interesting map $R$ from the projector to a rotated projector given by:

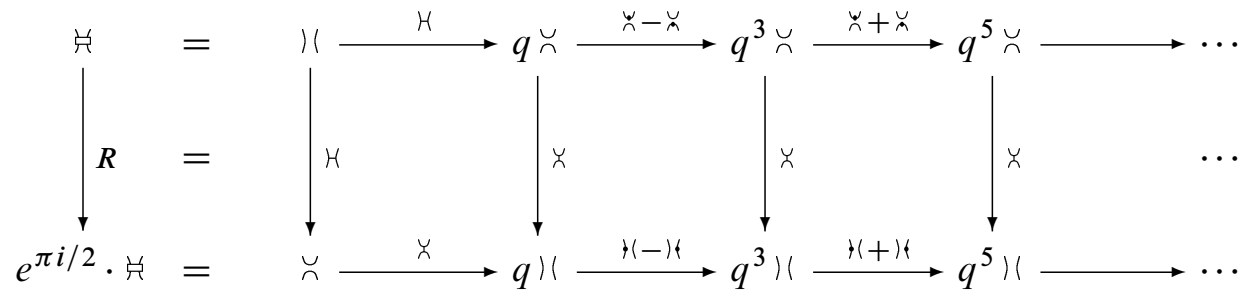

The square $R^{2}$ is a map from the projector to itself which, by neck-cutting, is equal to 总 + 兑. This is homotopic to zero by the above discussion. In fact

$$
R^{2}=d h+h d,
$$


where

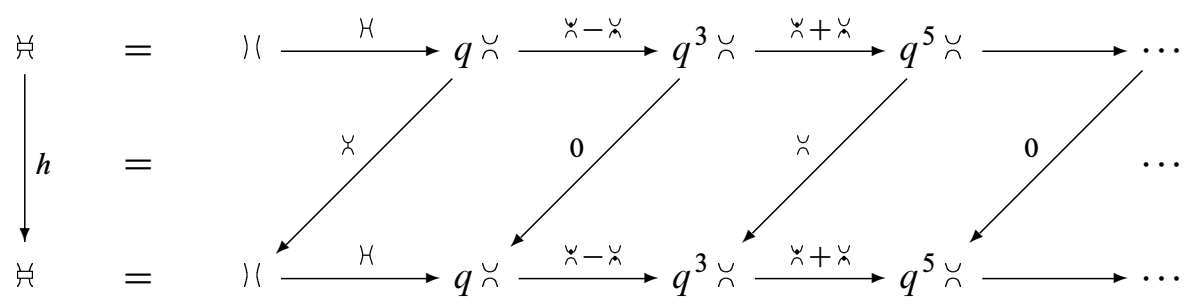

and maps alternate between 0 and $乞$. Together, the maps $R$ and $h$ can be used to construct a new differential on the complex formed by pairing a planar graph $G$ with its dual $\widehat{G}$.

\subsection{A structural conjecture}

A cube complex $C=\bigoplus_{v \in\{0,1\}^{n}} C_{v}$ is a chain complex of diagrams $C_{v}$ indexed by the vertices of a hypercube $\{0,1\}^{n}$. For any vertex $v \in\{0,1\}^{n}$ set $|v|=\sum_{i} v_{i}$. For any two vertices $v=\left(v_{1}, \ldots, v_{n}\right)$ and $w=\left(w_{1}, \ldots, w_{n}\right)$ we say $v \leq w$ if $v_{i} \leq w_{i}$ where $1 \leq i \leq n$. If $C$ is a cube complex and $v$ is a vertex then we define the star of $v$ in $C$, $\mathrm{St}_{v}(C) \subset C$, to be the subcomplex

$$
\mathrm{St}_{v}(C)=\bigoplus_{v \leq w} C_{w}
$$

Conjecture For every connected planar graph $G$ there exists a cube complex $C=$ $\bigoplus_{v \in\{0,1\}^{n}} C_{v}$ such that

$$
\langle G\rangle \simeq \bigoplus_{v \in\{0,1\}^{n}}\left(\frac{t^{2} q^{3}}{1-t^{2} q^{4}}\right)^{|v|} \cdot \operatorname{Cone}^{|v|}\left(\operatorname{St}_{v}(C) \stackrel{\sigma}{\rightarrow} q^{2} \operatorname{St}_{v}(C)\right)
$$

where the map $\sigma$ is a handle.

In other words, every chain complex breaks up into a direct sum of subcomplexes most of which are iterated cones on handle maps. This is precisely what happens in the computation for the theta graph in Section 7.2.

\subsection{Structure of the knot invariant}

In this section we will discuss the structure of the knot invariant defined in Section 5. 
Interesting homology is concentrated in low degree For any knot the homology defined in Section 5 is necessarily infinitely generated. However for any two knots we will show that all but a finite portion of this homology is the same, and the interesting part in low degree is closely related to the Khovanov categorification of the 2-colored Jones polynomial; see the discussion following the proof of Corollary 8.6.

Recall that in Section 3 the dot map was defined in terms of a handle and the differential $d_{n}$ of $P_{2}$ for $n>0$ was defined in Section 4.1 using sums and differences of these dot maps. The proposition below implies that these maps do not change up to sign and homotopy under the "dotted second Reidemeister move".

8.5 Proposition (Handles slide through crossings.)

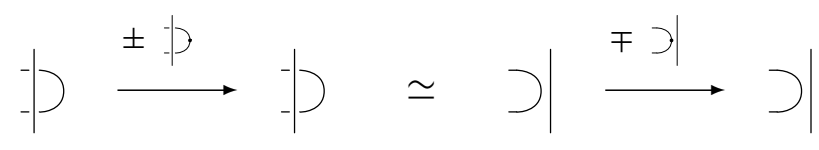

The proof follows from applying the Gaussian Elimination (Section 3.2) twice on the cube obtained by expanding the crossings on the left hand side above.

8.6 Corollary The chain complex associated to a framed knot $K$ in Section 5 is homotopy equivalent to

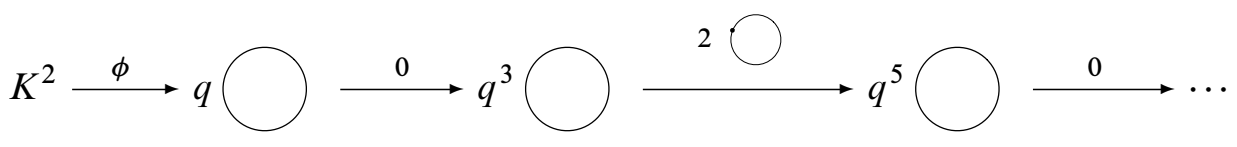

where $K^{2}$ denotes the 2-cabling of the knot $K$, and the map $\phi$ is induced by the homotopy.

Proof The first differential in $P_{2}$ (Section 4.1) is a saddle map which turns $K^{2}$ into the unknot. Using Proposition 8.5 (applying the Gaussian elimination to the chain complex for $K$ ) one slides the end of this unknotted 2-cabling through the rest of the knot. The result is pictured above. Note that the map $\phi$ in the statement of the corollary is a saddle map followed by a sequence of Reidemeister type II equivalences.

A similar statement may be proved for any link $L$. However, the infinite tail for knots, pictured in Corollary 8.6, is standard. When the number of components of $L$ is greater than one this infinite tail will involve chain complexes for the proper sublinks of $L$. 
Relationship to Khovanov's categorification A categorification of the colored Jones polynomials was given in [4]. When $n=2$ this construction coincides with the one in Section 5. Here we discuss the relationship between the categorification above and Khovanov's categorification of the colored Jones polynomial [12] when $n=2$.

Khovanov defines a chain complex

$$
C_{\mathrm{Kh}}(K)=\operatorname{Cone}\left(K^{2} \stackrel{\epsilon_{*}}{\rightarrow} \varnothing\right)
$$

which categorifies the 2-colored Jones polynomial of a framed knot $K . K^{2}$ is the chain complex which computes the Khovanov homology of the 2-cabling of $K$ and $\epsilon_{*}$ is induced by the 4 -dimensional cobordism $\epsilon: K^{2} \rightarrow \varnothing$ obtained by pushing the ribbon bounded by the 2 -cabling into the 4 -ball.

In order to define $\epsilon_{*}$ a Morse decomposition of $\epsilon$ must be chosen. Choose the one in which $\epsilon$ is a composition of a saddle followed by a disk bounding the resulting unknot. This is an augmentation of the first two terms of the chain complex in Corollary 8.6. If we denote these first two terms by $C_{\text {trunc }}(K)$ then there is a short exact sequence

$$
0 \rightarrow t q^{2} \mathbb{Z} \rightarrow C_{\text {trunc }}(K) \rightarrow C_{\mathrm{Kh}}(K) \rightarrow 0,
$$

where $t q^{2} \mathbb{Z}$ is the chain complex consisting only of $\mathbb{Z}$ in bidegree $(1,2)$. The associated long exact sequence implies that

$$
0 \rightarrow H_{\text {trunc }}^{0}(K) \rightarrow H_{\mathrm{Kh}}^{0}(K) \rightarrow q^{2} \mathbb{Z} \rightarrow H_{\text {trunc }}^{1}(K) \rightarrow H_{\mathrm{Kh}}^{1}(K) \rightarrow 0
$$

and $H_{\text {trunc }}^{i}(K) \cong H_{\mathrm{Kh}}^{i}(K)$ for $i \neq 0,1$.

\section{Appendix: Computations for graphs and links}

The homology is given for certain families of graphs, and for some examples of links.

\subsection{Chromatic homologies of trees and cycles}

Let $\theta_{n}$ denote the $n$-th theta graph, with two vertices and $n$ edges connecting them. The graph $\theta_{n}$ is dual to a cycle with $n$ edges or the boundary of an $n$-gon (see illustration in Section 6.1). The graph $T_{n}$ is dual to the graph with a single vertex and $n$ loops. The chain complex $\left\langle T_{n}\right\rangle$ computes the chromatic homology of any tree with $n$ edges. The homology of the trace $T_{1}=\operatorname{tr}\left(P_{2}\right)$ (Section 7.1) and the chain complex $E$ (Section 7.2) are used below to express the homologies of $T_{n}$ and $\theta_{n}$. 

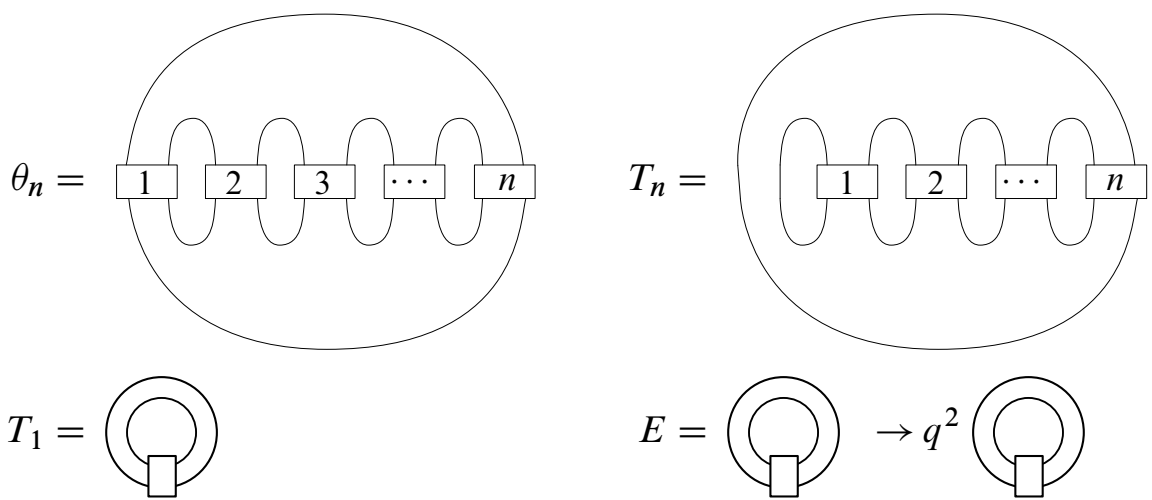

Using $\mathbb{Z}$ coefficients and $\alpha=0$ we have

$$
\begin{aligned}
& H\left(T_{n}\right)=\left.q^{1-n} H\left(T_{1}\right) \oplus \frac{q^{1-n}}{1+x}\left(\left(1+\frac{x^{2}}{1-x}\right)^{n-1}-1\right]\right|_{x=t q^{2}} \cdot H(E), \\
& H\left(\theta_{n}\right)=H\left(B_{n}\right) \oplus \frac{q^{2-n}}{1-x^{2}}\left(\left(1+\frac{x^{2}}{1-x}\right)^{n-1}\right. \\
& \left.\quad-\left(x+\frac{x^{2}}{1-x}\right)^{n-1}+x^{n-1}-1\right)\left.\right|_{x=t q^{2}} \cdot H(E),
\end{aligned}
$$

where

$$
\begin{aligned}
& H\left(B_{n}\right)= \begin{cases}\left.q^{1-2 k} \frac{1-x^{2 k}}{1-x^{2}}\right|_{x=t q^{2}} \cdot H(E) & \text { for } n=2 k+1, \\
\left.q^{2-2 k} H\left(T_{1}\right) \oplus q^{2-2 k} \frac{x-x^{2 k-1}}{1-x^{2}}\right|_{x=t q^{2}} \cdot H(E) & \text { for } n=2 k,\end{cases} \\
& H(E)=\left(q^{-2}+t q^{2}+t^{2} q^{2}+\frac{t^{3}\left(q^{4}+q^{6}\right)}{1-t q^{2}}\right) \cdot \mathbb{Z} \oplus\left(t+\frac{t^{3} q^{4}}{1-t q^{2}}\right) \cdot \mathbb{Z} / 2, \\
& H\left(T_{1}\right)=\left(q^{-2}+1+\frac{t^{2} q^{2}+t^{3} q^{6}}{1-t^{2} q^{4}}\right) \cdot \mathbb{Z} \oplus\left(\frac{t^{3} q^{4}}{1-t^{2} q^{4}}\right) \cdot \mathbb{Z} / 2 .
\end{aligned}
$$

\subsection{Knots and links}

If $2_{1}^{2}, 3_{1}$ and $4_{1}$ denote the Hopf link, the positively oriented trefoil and figure eight knots respectively then their homologies have been computed:

$$
\begin{aligned}
H\left(2_{1}^{2}\right)= & \left(t^{-4}\left(q^{-8}+q^{-6}\right)+t^{-2} q^{-4}+t^{-1}+\left(1+q^{-2}\right)+t\left(1+q^{2}+q^{4}\right)\right. \\
& \left.+t^{2} q^{4}+t^{4}\left(q^{6}+q^{8}\right)\right) \cdot \mathbb{Z}+\left(t^{-1} q^{-2}+q^{2} t^{2}\right) \cdot \mathbb{Z} / 2 \\
& +H\left(T_{1}\right)^{2}-\left(q^{-2}+1\right)^{2} \cdot \mathbb{Z}
\end{aligned}
$$




$$
\begin{aligned}
& H\left(3_{1}\right)=\left(t^{-6}\left(q^{-10}+q^{-8}\right)+t^{-4} q^{-6}+t^{-3} q^{-2}+t^{-2}\left(q^{-4}+q^{-2}\right)\right. \\
& +t^{-1}\left(1+q^{2}\right)+\left(1+q^{-2}\right)+t\left(q^{2}+q^{4}\right)+t^{2} q^{2}+t^{3} q^{6}+t^{5} q^{8} \\
& \left.+t^{6} q^{12}\right) \cdot \mathbb{Z} \\
& +\left(t^{-3} q^{-4}+t\left(1+q^{2}\right)+t^{3}\left(2 q^{4}+q^{6}\right)+t^{4}\left(q^{6}+q^{8}\right)+t^{6} q^{10}\right) \cdot \mathbb{Z} / 2 \\
& +H\left(T_{1}\right)-\left(q^{-2}+1\right) \cdot \mathbb{Z} \\
& H\left(4_{1}\right)=\left(t^{-8} q^{-14}+t^{-7} q^{-10}+t^{-5} q^{-8}+t^{-4}\left(q^{-8}+q^{-4}\right)+t^{-3}\left(q^{-6}+q^{-4}\right)\right. \\
& +t^{-2}\left(q^{-6}+q^{-4}+q^{-2}\right)+t^{-1}\left(q^{-4}+2 q^{-2}+1\right)+\left(2 q^{-2}+3+q^{2}\right) \\
& +t\left(1+2 q^{2}+q^{4}\right)+t^{2}\left(q^{2}+q^{4}+q^{6}\right)+t^{3}\left(q^{4}+q^{6}\right)+t^{4}\left(q^{4}+q^{8}\right) \\
& \left.+t^{5} q^{8}+t^{7} q^{10}+t^{8} q^{14}\right) \cdot \mathbb{Z} \\
& +\left(t^{-7} q^{-12}+t^{-5}\left(q^{-10}+q^{-8}\right)+t^{-4}\left(q^{-8}+2 q^{-6}\right)+t^{-3} q^{-6}\right. \\
& +t^{-2}\left(2 q^{-4}+q^{-2}\right)+t^{-1}\left(q^{-4}+2 q^{-2}\right)+\left(q^{-2}+1\right)+t\left(1+q^{2}\right) \\
& +t^{2}\left(1+2 q^{2}+q^{4}\right)+t^{3}\left(q^{2}+2 q^{4}\right)+t^{4} q^{6}+t^{5}\left(2 q^{6}+q^{8}\right) \\
& \left.+t^{6}\left(q^{8}+q^{10}\right)+t^{8} q^{12}\right) \cdot \mathbb{Z} / 2 \\
& +H\left(T_{1}\right)-\left(q^{-2}+1\right) \cdot \mathbb{Z}
\end{aligned}
$$

The $H\left(T_{1}\right)-\left(q^{-2}+1\right) \cdot \mathbb{Z}$ term is the infinite tail; see Section 8.4. Notice in $4_{1}$ that the free part of the homology is symmetric away from homological degree 0 . The missing $q^{2}$ term can be found in homological degree 2 of the infinite tail, giving a symmetric graded Euler characteristic. This was computed using the JavaKh program written by Jeremy Green and Scott Morrison [8].

Acknowledgements B Cooper wishes to thank Justin Roberts for many conversations on the subject of the paper.

The authors would like to thank the referee for a number of suggestions which have led to a better exposition.

V Krushkal was supported in part by NSF grant DMS-1007342 and by the IHES.

\section{References}

[1] D Bar-Natan, Khovanov's homology for tangles and cobordisms, Geom. Topol. 9 (2005) 1443-1499 MR2174270

[2] D Bar-Natan, Fast Khovanov homology computations, J. Knot Theory Ramifications 16 (2007) 243-255 MR2320156 
[3] J S Birman, H Wenzl, Braids, link polynomials and a new algebra, Trans. Amer. Math. Soc. 313 (1989) 249-273 MR992598

[4] B Cooper, V Krushkal, Categorification of the Jones-Wenzl projectors arXiv: 1005.5117

[5] P Fendley, V Krushkal, Link invariants, the chromatic polynomial and the Potts model, Adv. Theor. Math. Phys. 14 (2010) 507-540 MR2721654

[6] P Fendley, N Read, Exact $S$-matrices for supersymmetric sigma models and the Potts model, J. Phys. A 35 (2002) 10675-10704 MR1959217

[7] I Frenkel, C Stroppel, J Sussan, Categorifying fractional Euler characteristics, JonesWenzl projector and $3 j$-symbols with applications to Exts of Harish-Chandra bimodules arXiv:1007.4680

[8] J Green, S Morrison, JavaKh, Knot atlas (2008) Available at http://http:// katlas.org/svn/KnotTheory/tags/stable/src/Kh.m

[9] L Helme-Guizon, Y Rong, A categorification for the chromatic polynomial, Algebr. Geom. Topol. 5 (2005) 1365-1388 MR2171813

[10] L H Kauffman, P Vogel, Link polynomials and a graphical calculus, J. Knot Theory Ramifications 1 (1992) 59-104 MR1155094

[11] M Khovanov, A functor-valued invariant of tangles, Algebr. Geom. Topol. 2 (2002) 665-741 MR1928174

[12] M Khovanov, Categorifications of the colored Jones polynomial, J. Knot Theory Ramifications 14 (2005) 111-130 MR2124557

[13] J Murakami, The Kauffman polynomial of links and representation theory, Osaka J. Math. 24 (1987) 745-758 MR927059

[14] L Rozansky, An infinite torus braid yields a categorified Jones-Wenzl projector arXiv: 1005.3266

[15] M Stošić, New categorifications of the chromatic and dichromatic polynomials for graphs, Fund. Math. 190 (2006) 231-243 MR2232861

[16] CA Weibel, An introduction to homological algebra, Cambridge Studies in Advanced Math. 38, Cambridge Univ. Press (1994) MR1269324

[17] S Yamada, An operator on regular isotopy invariants of link diagrams, Topology 28 (1989) 369-377 MR1014466

Department of Mathematics, University of Virginia

Charlottesville VA 22904-4137, USA

bjc4n@virginia.edu, mhoganca@gmail.com, krushkal@virginia.edu http://people.virginia.edu/ bjc4n, http://www.math.virginia.edu/ vk6e

Received: 29 December 2010 